\title{
Lost in Translation: Topological Singularities in Group Field Theory
}

\author{
Razvan Gurau*
}

October 23, 2018

\begin{abstract}
Random matrix models generalize to Group Field Theories (GFT) whose Feynman graphs are dual to gluings of higher dimensional simplices. It is generally assumed that GFT graphs are always dual to pseudo manifolds. In this paper we prove that already in dimension three (and in all higher dimensions), this is not true due to subtle differences between simplicial complexes and gluings dual to GFT graphs.

We prove however that, fortunately, the recently introduced "colored" GFT models [1] do not suffer from this problem and only generate graphs dual to pseudo manifolds in any dimension.
\end{abstract}

\section{Introduction: Group Field Theory}

Group Field Theories (GFT) [2, 3] are quantum field theories over group manifolds. They generalize random matrix models and random tensor models [4, 5. (see also [6, 7]). GFT's arise naturally in several discrete approaches to quantum gravity, like Regge calculus [8], dynamical triangulations [9] or spin foam models [10] (see [11] for further details).

The Feynman graphs of GFT are built from vertices encoding the connectivity dual to a $n$ simplex, and propagators encoding the connectivity dual to the gluing of $n$ simplices along boundary $(n-1)$ simplices. A graph is dual to a "gluing of simplices" yielding some $n$ dimensional topological space,

${ }^{*}$ Perimeter Institute for Theoretical Physics Waterloo, ON, N2L 2Y5, Canada 
and GFT's generalize the familiar matrix models [12] to a theory of random higher dimensional topological spaces.

In discrete approaches to quantum gravity [11, 13], a gluing of simplices is interpreted as a space-time background making GFT a combinatorial, background independent theory, whose perturbative development generates space-times. This is further supported as, for the simplest choice of vertex and propagator, the Feynman amplitude of a graph reproduces the partition function of a BF theory discretized on the gluing of simplices [2, 14]1. BF theory becomes Einstein gravity after implementing the Plebanski constraints and it is natural to suppose that a some more involved GFT model will reproduces the partition function of the latter. Working at the level of individual GFT graphs (spin foams), one has a good control over the constraints, and their implementation leads to several alternative propositions ([18, 19] or [20, 21]) of vertex kernels. The semiclassical limit [22, 23] of these models has been analyzed with encouraging results. Alternatively, one can try to implement the constraints directly at the level of the action $([24,25]$ or [26]) or include matter fields [27, 28]. Recently GFT's and spin foams have been adapted to the study of loop quantum cosmology [29, 30].

Irrespective of the particularities of the model, the fundamental question in all discrete approaches to quantum gravity is "to sum or not to sum?". According to the answer to this question one distinguishes several possibilities. Spin foam models sum over all metrics at fixed triangulation, dynamical triangulations sum over subclasses of triangulations 2 at fixed topology, while GFT's sum over everything. The weights of different topologies, triangulations and metrics are completely fixed by the Feynman rules. In two dimensions GFT's reduce to matrix models some of which [31, 32] are ultraviolet complete [33, 34]. This opens up the tantalizing possibility that the GFT's themselves are consistent and complete quantum field theories.

The scenario of GFT as a fundamental quantum field theory recently received renewed attention. Partial power counting theorems and bounds 35 , 36] have been obtained for the simplest GFT models. More accurate power counting theorems have been established [37, 38] for the "colored GFT's" [1, 39] and recently extended [40].

However there is a fundamental aspect of GFT's which has been little

\footnotetext{
${ }^{1}$ In algebraic combinatorics this lead to new topologycal invariants [15] and advances on the volume conjecture [16, 17.

${ }^{2}$ With metric fixed for a given triangulation.
} 
addressed so far but has the potential to completely invalidate them: the topology of the gluings dual to GFT graphs. It hes been noted from some time [41] that GFT's generate not only manifolds but also pseudo manifolds. As it is clear that space time is a manifold, this is a rather unpleasant feature of GFT's. However it is not critical: pseudo manifolds are related (one to one) to manifolds with boundary, and gravity makes perfect sense on the latter. In fact in at least an approach to spin foams (and consequently GFT) such configurations are desirable, [42]: the topological defects of pseudo manifolds can be interpreted as matter coupled to the gravitational background.

This paper addresses, in its full generality, the problem of the topology of gluings dual to GFT graphs. An in depth study of this question will reveal a very serious issue which has been largely ignored up to now: in all dimensions, including three, there exist GFT graphs dual to gluings which are not pseudo manifolds but correspond to much more singular topologies. The simplest example of such a graph is presented in figure1. We will present

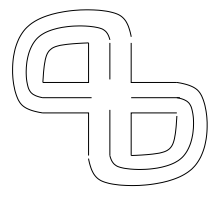

Figure 1: A graph not dual to a pseudo manifold.

in detail this example in section 4 , but for now it suffices to say that the Euler characteristic of its dual gluing is -1 . It is a fundamental result (which we recall in section 2 for completeness) that the Euler characteristic of three dimensional pseudo manifolds is allways greater or equal to zero, thus this graph can not correspond to a pseudo manifold.

The pathological singularities we identify in this paper are generic, appearing at arbitrary order in perturbations, and dominate in power counting. This brings into question the usual GFT's status as "fundamental" quantum field theories: their effective behavior is dominated by pathological configurations. In retrospect, when compared to these pathologies, the pseudo manifolds seem just a small nuisance one can live with.

However GFT's miss their target only by an inch. If one assumes that the gluing dual to a GFT graph is a simplicial complex 3 , then all the pathologies disappear and the gluing is a pseudo manifold.

\footnotetext{
${ }^{3}$ Or that it becomes one after subdivision.
} 
In order to salvage the GFT's as quantum field theories one must find some way to eliminate the singular topologies. Restricting, by some condition at the level of the action the allowed gluings one can hope that only pseudo manifolds are created. However, finding a good restriction is a subtle question. For instance, requiring that the identification of two $(n-1)$ simplices respects orientations is largely insufficient: their $(n-2),(n-3)$ etc. subsimplices are identified in a completely arbitrary way and generate pathologies.

A surprisingly simple solution to this problem is provided by the recently introduced "colored" GFT (CGFT) models . This model completely eliminates all the pathologies, yielding only pseudo manifolds in any dimension by an unique prescription. Establishing this result is the "raison d'être" of this paper. We will prove this in all the technical detail in section 5, but the

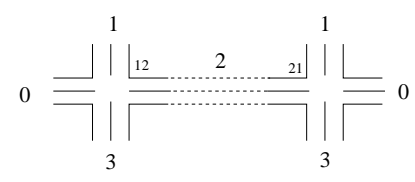

Figure 2: Colored GFT line.

profound reason which makes the colored models work is very intuitive. If we denote a strand (solid line in figure 2) by the colors of the two halflines to which it belongs, the colored GFT lines will allways conserve the labels of the strands. In turn this will guarantee that all subsimplices (of any dimension) are identified respecting their orientations. In retrospect the colored prescription is very natural: it is the simplest one which ensures this. We view this result as a very strong argument in support of the colored GFT models.

This paper is organized as follows. In section 2 we review some definitions and classical results concerning normal simplicial pseudo manifolds. In section 3 we describe in detail the usual GFT's and their graphs, and introduce the link graphs in subsection 3.1. We detail at length the pathological wrapping singularities plaguing the usual GFT's in section 4. In section 5 we recall the colored GFT models and prove that they only generate graphs dual to normal simplicial pseudo manifolds. Finally, in section 6, we review the implications of our result. We will only deal with closed GFT graphs, the generalization to open graphs [39] being immediate. 


\section{Simplicial Pseudo Manifolds}

In this section we review some definitions and properties of normal simplicial pseudo manifolds (following the notations of [43]) relevant to our subsequent analysis of GFT's.

A finite abstract simplicial complex is a finite set $A$ together with a collection $\Delta$ of subsets such that if $X \in \Delta$ and $Y \subseteq X$ then $Y \in \Delta$.

An element $v \in A$ such that $\{v\} \in \Delta$ is called a vertex of $\Delta$, and the set of all vertices of $\Delta$ is denoted $V(\Delta)$. An element $\sigma \in \Delta$ is called a simplex. The proper subsets $\tau$ of a simplex $\sigma,(\tau \subset \sigma, \sigma \backslash \tau \neq \emptyset)$ are called faces or subsimplices of $\sigma$. Note that $\Delta$ is not a set but a collection (or a multiset), meaning that the same simplex can appear several times in $\Delta$. A subcomplex $\Delta^{\prime}$ of $\Delta$ is a simplicial complex such that $\sigma \in \Delta^{\prime} \Rightarrow \sigma \in \Delta$. To any simplex in a simplicial complex one canonically associates several simplicial subcomplexes of $\Delta$

- The deletion of $\tau$ is the abstract simplicial subcomplex of $\Delta$

$$
\mathrm{dl}_{\Delta}(\tau)=\{\sigma \in \Delta \mid \tau \nsubseteq \sigma\}
$$

- The link of $\tau$ is the abstract simplicial subcomplex of $\Delta$

$$
\operatorname{lk}_{\Delta}(\tau)=\{\sigma \in \Delta \mid \sigma \cap \tau=\emptyset \text { and } \sigma \cup \tau \in \Delta\} \text {. }
$$

- The closed star of $\tau$ is the abstract simplicial subcomplex of $\Delta$

$$
\operatorname{star}_{\Delta}(\tau)=\{\sigma \in \Delta \mid \sigma \cup \tau \in \Delta\} .
$$

The link and the closed star of a simplex $\tau$ are related by

$$
\operatorname{lk}_{\Delta}(\tau)=\operatorname{star}_{\Delta}(\tau) \cap \mathrm{dl}_{\Delta}(V(\tau))
$$

as $\sigma \nsupseteq\{v\}, \forall v \in V(\tau) \Rightarrow \sigma \cap \tau=\emptyset$.

For any vertex $v$ of $\Delta$, and any simplex $\sigma \in \Delta$, either $\{v\} \nsubseteq \sigma$ or $\{v\} \cup \sigma=\sigma \in \Delta$, hence

$$
\Delta=\operatorname{star}_{\Delta}(v) \cup \mathrm{dl}_{\Delta}(v) .
$$

\footnotetext{
${ }^{4}$ Or simplicial complex, for brevity.
} 
A simplex $\tau$ of a simplicial complex $\Delta$ has dimension $n$ (it is an $n$ simplex) if it has cardinality $n+1$. For instance, the vertices of $\Delta$ have dimension 0 . We denote the number of simplices of dimension $p$ in $\Delta$ by $f^{p}(\Delta)$ (hence $\left.f^{0}(\Delta)=|V(\Delta)|\right)$ and its Euler characteristic by

$$
\chi(\Delta)=\sum_{p \geq 0}(-1)^{p} f^{p}(\Delta) .
$$

For any vertex $v$ eq. (4) and (5) imply that the Euler characteristic of a simplicial complex respects

$$
\begin{aligned}
\chi(\Delta) & =\chi\left(\operatorname{star}_{\Delta}(v)\right)+\chi\left(\operatorname{dl}_{\Delta}(v)\right)-\chi\left(\operatorname{lk}_{\Delta}(v)\right) \\
& =1-\chi\left(\operatorname{lk}_{\Delta}(v)\right)+\chi\left(\operatorname{dl}_{\Delta}(v)\right),
\end{aligned}
$$

where in the last line we used $\chi\left(\operatorname{star}_{\Delta}(v)\right)=1$ (see appendix A $)$.

An $n$-dimensional simplicial pseudo manifold is a finite abstract simplicial complex with the following properties:

- it is non-branching: Each $(n-1)$ simplex is a face of precisely two $n$ simplices.

- it is strongly connected: Any two $n$ simplices can be joined by a "strong chain" of $n$ simplices in which each pair of neighboring simplices have a common $(n-1)$ simplex.

- it is pure (it has dimensional homogeneity): Each simplex is a face of some $n$ simplex.

A pseudo manifold is called normal if all its links are pseudo manifolds. This condition can fail (see appendix A] because the links of a pseudo manifold, while always being pure, non branching simplicial complexes, are not in general strongly connected. Crucial in the sequel is the following property of three dimensional normal pseudo manifolds

Proposition 1. The Euler character of a three dimensional normal pseudo manifold $\Delta$ respects

$$
\chi(\Delta)=|V(\Delta)|-\frac{1}{2} \sum_{i} \chi\left(l k_{\Delta}\left(v_{i}\right)\right),
$$

and $\chi\left(l k_{\Delta}\left(v_{i}\right)\right) \leq 2$, thus $\chi(\Delta) \geq 0$. 
Proof: Counting subsets of fixed cardinality shows that a pure simplicial complex respect

$$
\begin{aligned}
& \sum_{i} f^{2}\left(\operatorname{lk}_{\Delta}\left(v_{i}\right)\right)=4 f^{3}(\Delta) \\
& \sum_{i} f^{1}\left(\operatorname{lk}_{\Delta}\left(v_{i}\right)\right)=3 f^{2}(\Delta) \\
& \sum_{i} f^{0}\left(\operatorname{lk}_{\Delta}\left(v_{i}\right)\right)=2 f^{1}(\Delta) .
\end{aligned}
$$

In a non branching simplicial complex in three dimensions every 3 simplex is bounded by four 2 simplices and every 2 simplex belongs to exactly two 3 simplices, hence

$$
4 f^{3}(\Delta)=2 f^{2}(\Delta)
$$

hence the Euler characteristic of a three dimensional pure, non branching simplicial complex respects

$$
\chi(\Delta)=f^{0}(\Delta)-\frac{1}{2} \sum_{i} \chi\left(\operatorname{lk}_{\Delta}\left(v_{i}\right)\right) .
$$

If, furthermore, $\Delta$ is a normal pseudo manifold, all the links of its vertices are two dimensional pseudo manifolds. A link, $\mathrm{lk}_{\Delta}\left(v_{i}\right)$, is strongly connected, hence there exists a "strong tree" of 1 simplices connecting all its 2 simplices. If one deletes the 1 simplices in the strong tree (and glues the 2 simplices into a patch), the 0 simplices are still connected by (at least a tree of) the remaining 1 simplices, thus

$$
f^{1}\left(\mathrm{lk}_{\Delta}\left(v_{i}\right)\right) \geq\left[f^{2}\left(\mathrm{lk}_{\Delta}\left(v_{i}\right)\right)-1\right]+\left[f^{0}\left(\mathrm{lk}_{\Delta}\left(v_{i}\right)\right)-1\right],
$$

which achieves the proof.

\section{GFT Graphs}

In this section we detail the Feynman graphs of the usual GFT models in $n$ dimensions and relate them with normal simplicial pseudo manifolds. 
The usual $n$ dimensional GFT model is defined for a scalar field $\phi$ : $G^{n} \rightarrow \mathbb{R}$, (with $G$ some Lie group), symmetric under permutations $\pi$ of its arguments and invariant under simultaneous left multiplication

$$
\begin{aligned}
& \phi\left(g_{\alpha_{\pi(1)}}, \ldots, g_{\alpha_{\pi(n)}}\right)=\phi\left(g_{\alpha_{1}}, \ldots, g_{\alpha_{n}}\right), \forall \pi \text { permutation }, \\
& \phi\left(h g_{\alpha_{1}}, \ldots, h g_{\alpha_{n}}\right)=\phi\left(g_{\alpha_{1}}, \ldots, g_{\alpha_{n}}\right), \forall h \in G .
\end{aligned}
$$

The GFT action in $n$ dimensions is [41]

$$
\begin{aligned}
S= & \frac{1}{2} \int[d g] \phi_{\alpha_{0} \alpha_{1} \ldots \alpha_{n}} \phi_{\alpha_{0} \alpha_{1} \ldots \alpha_{n}}+S_{i n t}, \\
S_{i n t}= & \frac{\lambda}{n+1} \int[d g] \phi_{\alpha_{0 n} \alpha_{0 n-1} \ldots \alpha_{01}} \ldots \phi_{\alpha_{p p-1} \ldots \alpha_{p 0} \alpha_{p n} \ldots \alpha_{p p+1}} \\
& \times \ldots \phi_{\alpha_{n n-1} \ldots \alpha_{n 0}}
\end{aligned}
$$

where $\phi_{\alpha_{0} \alpha_{1} \ldots \alpha_{n}} \equiv \phi\left(g_{\alpha_{0}}, g_{\alpha_{1}}, \ldots, g_{\alpha_{n}}\right)$, and $g_{\alpha_{i j}}=g_{\alpha_{j i}}$ in $S_{i n t}$. The GFT vertex generated by $S_{i n t}$, is represented in figure 3 .

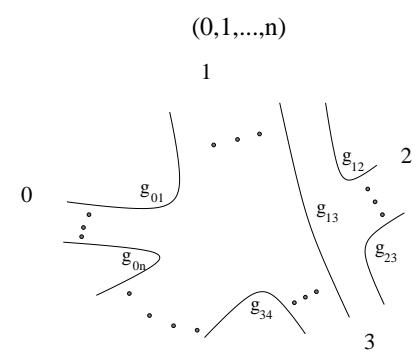

Figure 3: GFT vertex in $n$ dimensions.

Each field $\phi$ in $S_{\text {int }}$ is associated to a half line of the GFT vertex. Every two fields in $S_{i n t}$ share a group element, consequently every two half lines of the GFT vertex share a strand (depicted as a solid line in figure 3). We label the half lines of the GFT vertex 0,1 up to $n$ and each strand by the (unordered) couple of labels of the two half lines which share it (that is the strand $i j$ is shared by the half lines $i$ and $j$ ).

The GFT vertex $(0 \ldots n)$ is dual to an $n$ simplex $\left\{A_{0}, \ldots A_{n}\right\}$. The half lines of the vertex represent the $(n-1)$ simplices bounding the $n$ simplex, namely the half line $i$ represents the simplex opposite to the vertex $A .5$.

$$
\left\{A_{0}, \ldots A_{n}\right\} \backslash\left\{A_{i}\right\} \equiv\left\{A_{0}, \ldots \widehat{A_{i}}, \ldots A_{n}\right\} .
$$

\footnotetext{
${ }^{5}$ Throughout this paper we denote by a hat the absence of a symbol in a list.
} 
The strand $i j$ represents the $(n-2)$ simplex shared by the two $(n-1)$ simplices $\left\{A_{0}, \ldots \widehat{A_{i}}, \ldots A_{n}\right\}$ and $\left\{A_{0}, \ldots \widehat{A_{j}}, \ldots A_{n}\right\}$, that is

$$
\left\{A_{0}, \ldots \widehat{A}_{i}, \ldots, \widehat{A}_{j}, \ldots A_{n}\right\}
$$

In the sequel the GFT vertex will be called a stranded vertex, to emphasize its internal strand structure.

We use this opportunity to clarify a somewhat confusing point: the half lines of GFT vertex are not dual to $(n-1)$ simplices, they are graphical representations. An $(n-1)$ simplex is dual to a GFT vertex in the appropriate dimension. This distinction is crucial in order to understand the link graphs of section 3.1

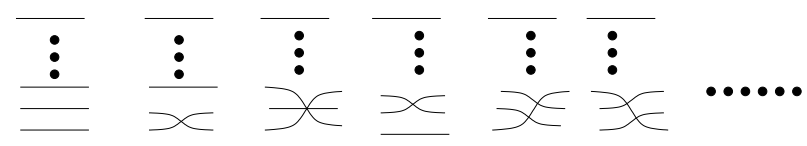

Figure 4: GFT lines in $\mathrm{n}$ dimensions.

The GFT propagator, generated by the quadratic part of the action (14), connects two GFT vertices via an arbitrary permutation of the strands. Some possible choices of GFT lines are presented in figure 4. The GFT lines represent the identification of two $(n-1)$ simplices and each permutation of the strands encodes one of the $n$ ! possible ways to do this. Like the GFT vertices, the GFT lines are stranded and have an internal structure.

To a GFT line $\ell_{v_{A} v_{B}}$ connecting two GFT vertices $v_{A}$ and $v_{B}$ (and oriented from $v_{A}$ to $v_{B}$ ) dual to the $n$ simplices

$$
\sigma_{A}^{n}=\left\{A_{0}, \ldots, A_{n}\right\}, \quad \sigma_{B}^{n}=\left\{B_{0}, \ldots, B_{n}\right\},
$$

we associate a function $\ell_{v_{A} v_{B}}:\{0, \ldots, n\} \rightarrow\{0, \ldots, n\}$ defined as follows. The line connects the half line $i$ of $v_{A}$ with some half line, say $k$, of $v_{B}$. We set $\ell_{v_{A} v_{B}}(i)=k$. Also, the line connects the strand $i j$ of $v_{A}$ to some strand, say $k l$ of $v_{B}$. We set $\ell_{v_{A} v_{B}}(j)=l$. The function $\ell_{v_{A} v_{B}}$ encodes the identification of the two $(n-1)$ simplices

$$
\left\{A_{0}, \ldots, \widehat{A_{i}}, \ldots A_{n}\right\},\left\{B_{0}, \ldots, \widehat{B_{F(i)}}, \ldots B_{n}\right\}
$$

and all their faces via $A_{j}=B_{F(j)}, \forall j \neq i$. 
The perturbative development of GFT is indexed by stranded Feynman graphs $\mathcal{G}$ generalizing the ribbon graphs of matrix models. A GFT graph $\mathcal{G}$ is dual to come gluing of $n$ simplices, denoted in the sequel $\Delta^{\mathcal{G}}$.

The gluing $\Delta^{\mathcal{G}}$ is a collection of $n$ simplices (and all their faces) modulo the identifications encoded in the lines. Clearly $X \in \Delta^{\mathcal{G}}$ and $Y \subset X$ then $Y \in \Delta^{\mathcal{G}}$, thus $\Delta^{\mathcal{G}}$ is very close to a simplicial complex. However, in general, $\Delta^{\mathcal{G}}$ is not a simplicial complex. Performing the identifications encoded in the lines one can end up identifying two a priori distinct vertices on the same $n$ simplex. Consequently, the elements $X \in \Delta^{\mathcal{G}}$ are not sets, but multiset: 6 .

This is not always a problem. It is possible that, even if $\Delta^{\mathcal{G}}$ is not a simplicial complex, it is still topologically equivalent to some simplicial complex $\tilde{\Delta}^{\mathcal{G}}$. What is much less obvious is that sometimes $\Delta^{\mathcal{G}}$ is not equivalent to any simplicial complex. This in turn leads to some very pathological singularities.

\subsection{Link graphs}

The links defined for simplicial complexes generalize immediately to gluings. The link of a $p$ simplex is a gluing of $(n-p-1)$ simplices, hence it is dual to a GFT graph in $(n-p-1)$ dimensions. We call this graph a link graph. To construct it, consider the $p$ simplex $\sigma^{p}=\left\{A_{i_{0}}, \ldots A_{i_{p}},\right\}$ in a gluing. The contribution of the $n$ simplex $\sigma^{n}=\left\{A_{0}, \ldots A_{n}\right\}$ to its link consist of the simplex $\sigma^{n-p-1}=\sigma^{n} \backslash \sigma^{p}$ and all its faces. The $n$ simplex $\sigma^{n}$ is dual to the GFT vertex $(0 \ldots n)$, therefore $\sigma^{n-p}$ is dual to the GFT vertex $\left(0 \ldots \widehat{i_{0}} \ldots \widehat{i_{p}} \ldots n\right)$ obtained by deleting all the half lines $i_{0}, \ldots i_{p}$ together with all their strands in the initial GFT vertex $(0, \ldots n)$. We call $\left(0 \ldots \widehat{i_{0}} \ldots \widehat{i_{p}} \ldots n\right)$ a descendant vertex of $(0 \ldots n)$. The $(n-p-1)$ link graphs are obtained by connecting the descendant $(n-p-1)$ vertices of all initial $n$ dimensional GFT vertices as dictated by the GFT lines.

Consider the example of three dimensional GFT whose vertex and dual three simplex (tetrahedron) are presented in figure 5 .

A vertex (say $A_{0}$ ) of the tetrahedron $\sigma^{3}=\left\{A_{0}, A_{1}, A_{2}, A_{3}\right\}$ is opposite to a triangle $\sigma^{2}=\sigma^{3} \backslash\left\{A_{0}\right\}=\left\{A_{1}, A_{2}, A_{3}\right\}$. This triangle is represented by a half line (the half line 0) in the GFT graph. Two triangles (say $\left\{A_{1}, A_{2}, A_{3}\right\}$ and $\left.\left\{A_{0}, A_{2}, A_{3}\right\}\right)$ share and edge on the tetrahedron (the edge $\left\{A_{2}, A_{3}\right\}$ ). This edge is represented by the strand common to the two half lines (the

\footnotetext{
${ }^{6}$ To add to the confusion recall that $\Delta$ itself is a multiset. Its elements $X \in \Delta$, however, must be sets.
} 


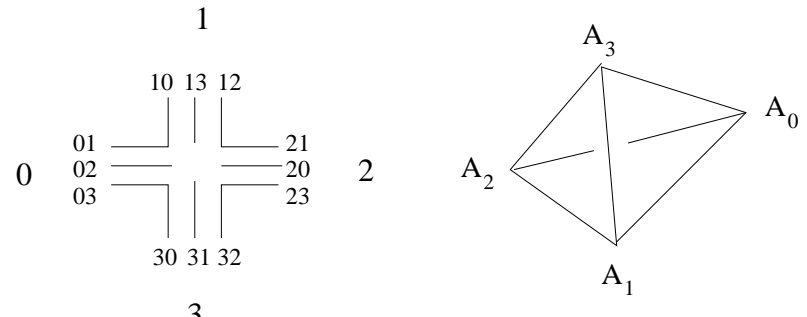

Figure 5: GFT vertex in 3 dimensions.

strand 01 , common to the half lines 0 and 1 ).

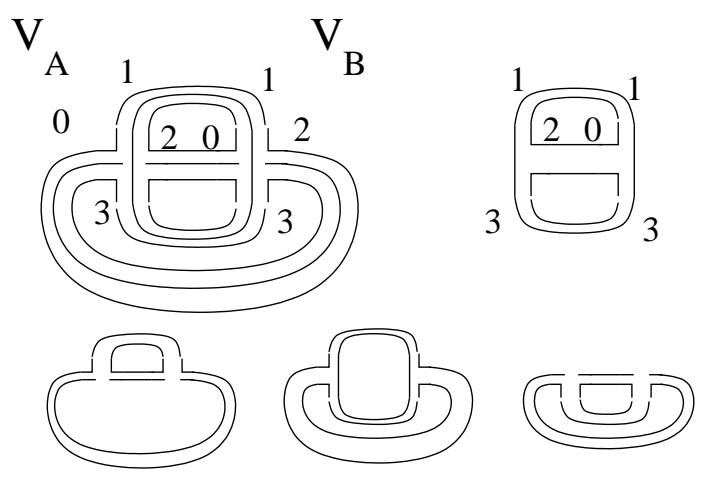

Figure 6: Link graphs in GFT.

Consider the example of the GFT graph in figure 6. Its dual gluing, $\Delta^{\mathcal{G}}$, consists of two tetrahedra $\left\{A_{0}, A_{1}, A_{2}, A_{3}\right\}$ and $\left\{B_{0}, B_{1}, B_{2}, B_{3}\right\}$, and four identifications (hence four functions) associated to the lines

$$
\begin{array}{ll}
\ell_{v_{A} v_{B}}^{(1)}(2)=0 ; & \ell_{v_{A} v_{B}}^{(1)}(1)=1, \ell_{v_{A} v_{B}}^{(1)}(0)=2, \ell_{v_{A} v_{B}}^{(1)}(3)=3, \\
\ell_{v_{A} v_{B}}^{(2)}(1)=1 ; & \ell_{v_{A} v_{B}}^{(2)}(2)=0, \ell_{v_{A} v_{B}}^{(2)}(3)=3, \ell_{v_{A} v_{B}}^{(2)}(0)=2, \\
\ell_{v_{A} v_{B}}^{(3)}(0)=2 ; & \ell_{v_{A} v_{B}}^{(3)}(1)=1, \ell_{v_{A} v_{B}}^{(3)}(2)=0, \ell_{v_{A} v_{B}}^{(3)}(3)=3, \\
\ell_{v_{A} v_{B}}^{(4)}(3)=3 ; & \ell_{v_{A} v_{B}}^{(4)}(0)=2, \ell_{v_{A} v_{B}}^{(4)}(1)=1, \ell_{v_{A} v_{B}}^{(4)}(2)=0,
\end{array}
$$

where the first column is the half line of $v_{A}$ from which each line originates, and the subsequent columns indicate the various identifications of $A$ 's with 
$B$ 's. The line $\ell_{v_{A} v_{B}}^{(1)}$ for example encodes he identifications

$$
\begin{aligned}
& \left\{A_{1}, A_{0}, A_{3}\right\}=\left\{B_{1}, B_{2}, B_{3}\right\} \quad\left\{A_{1}, A_{0}\right\}=\left\{B_{1}, B_{2}\right\} \\
& \left\{A_{1}, A_{3}\right\}=\left\{B_{1}, B_{3}\right\} \quad\left\{A_{0}, A_{3}\right\}=\left\{B_{2}, B_{3}\right\} \\
& \left\{A_{1}\right\}=\left\{B_{1}\right\} \quad\left\{A_{0}\right\}=\left\{B_{2}\right\} \quad\left\{A_{3}\right\}=\left\{B_{3}\right\} .
\end{aligned}
$$

The reader can convince himself that, after performing also the identifications corresponding to $\ell_{v_{A} v_{B}}^{(2)}, \ell_{v_{A} v_{B}}^{(3)}$ and $\ell_{v_{A} v_{B}}^{(4)}$, the gluing writes

$$
\begin{aligned}
\Delta^{\mathcal{G}}=\{ & \emptyset,\left\{A_{0}\right\},\left\{A_{1}\right\},\left\{A_{2}\right\},\left\{A_{3}\right\},\left\{A_{0}, A_{1}\right\},\left\{A_{0}, A_{2}\right\},\left\{A_{0}, A_{3}\right\}, \\
& \left\{A_{1}, A_{2}\right\},\left\{A_{1}, A_{3}\right\},\left\{A_{2}, A_{3}\right\},\left\{A_{0}, A_{1}, A_{2}\right\}, \\
& \left\{A_{0}, A_{1}, A_{3}\right\},\left\{A_{0}, A_{2}, A_{3}\right\},\left\{A_{1}, A_{2}, A_{3}\right\}, \\
& \left.\left\{A_{0}, A_{1}, A_{2}, A_{3}\right\},\left\{A_{0}, A_{1}, A_{2}, A_{3}\right\}\right\} .
\end{aligned}
$$

Note that $\Delta^{\mathcal{G}}$ is a multiset (the two 3 simplices have exactly the same vertices) and one can check that this gluing is a simplicial complex. The link of $A_{0}$, for instance writes

$$
\begin{aligned}
& \operatorname{lk}_{\Delta \mathcal{G}}\left(A_{0}\right)=\left\{\emptyset,\left\{A_{1}\right\},\left\{A_{2}\right\}\left\{A_{3}\right\},\left\{A_{1}, A_{2}\right\},\left\{A_{1}, A_{3}\right\},\left\{A_{2}, A_{3}\right\},\right. \\
& \left.\left\{A_{1}, A_{2}, A_{3}\right\},\left\{A_{1}, A_{2}, A_{3}\right\}\right\} \text {. }
\end{aligned}
$$

It is in fact easier to access directly the link graph dual to $\mathrm{lk}_{\Delta^{\mathcal{G}}}\left(A_{0}\right)$ starting from $\mathcal{G}$. To build the link graph dual to $\operatorname{lk}_{\Delta}\left(A_{0}\right)$ we distinguish the labels on the vertices $\mathcal{V}_{A}$ and $\mathcal{V}_{B}$ by a lower index. Take the descendant vertex $1_{A} 2_{A} 3_{A}$ obtained by deleting the half line $0_{A}$ (and all its strands) of $\left(0_{A} 1_{A} 2_{A} 3_{A}\right)$. The half lines of the descendant vertex inherit the labels of the corresponding GFT half lines, $1_{A}, 2_{A}$ and $3_{A}$, and a pair of descendant half lines share a strand $\left(1_{A}\right.$ and $2_{A}$ share the strand $1_{A} 2_{A}$, etc. $)$.

The half line $1_{A}$ of the descendant vertex $1_{A} 2_{A} 3_{A}$ connects to the half line $1_{B}$ of the descendant vertex $1_{B} 0_{B} 3_{B}$ (obtained by deleting $2_{B}$ ) of $0_{B} 1_{B} 2_{B} 3_{B}$. Similarly, the half line $2_{A}$ connects to $0_{B}$ and $3_{A}$ to $3_{B}$ of the same descendant vertex $1_{B} 0_{B} 3_{B}$. The two descendent's vertices thus form a connected graph, dual to the link $\operatorname{lk}_{\Delta}\left(A_{0}\right)$.

By construction, every GFT vertex in three dimensions has four descendants vertices in the link graphs, thus the dual gluing of any GFT graph respects

$$
\sum_{i} f^{2}\left(\mathrm{lk}_{\Delta}\left(v_{i}\right)\right)=4 f^{3}(\Delta)
$$


Also, any GFT line always has three descendants (any two strands of a GFT line yield a descendant line in some link graph), hence the dual gluing respects

$$
\sum_{i} f^{1}\left(\mathrm{lk}_{\Delta}\left(v_{i}\right)\right)=3 f^{2}(\Delta)
$$

Moreover each strand on the GFT vertex has two descendant strands in the link graphs.

The main result of this section is synthesized in the flowing lemma.

Lemma 1. If a gluing $\Delta^{\mathcal{G}}$ dual to a $n$ dimensional connected GFT graph $\mathcal{G}$ is a simplicial complex then it is a normal pseudo manifold.

Let us comment on this lemma before proving it. In the mathematical literature there are numerous results concerning pseudo manifolds (notoriously, for example, in three dimensions they only present isolated singularities). Whereas this results hold for some graphs, they fail in general. For instance, the Betti numbers and boundary operators, relevant for power counting estimates, can be defined only for graphs dual to pseudo manifolds. They make no sense for arbitrary GFT graphs.

Proof of lemma 1; A gluing dual to a connected GFT graph is always pure and strongly connected. The GFT lines either connect two different GFT vertices or are tadpole lines (they start and end on the same GFT vertex). Thus in the dual gluing the $(n-1)$ simplices either separate two distinct $n$ simplices or belong twice to the same $n$ simplex.

If a $(n-1)$ simplex belongs twice to the same $n$ simplex, then in its corresponding gluing at least two a priori distinct vertices of the $n$ simplex are identified. Thus the $n$ simplex is not represented by a set in $\Delta^{\mathcal{G}}$, but my a multiset and $\Delta^{\mathcal{G}}$ is not a simplicial complex. Consequently, if $\Delta^{\mathcal{G}}$ is a simplicial complex, then all its $(n-1)$ simplices bound exactly two $n$ simplices, therefore $\Delta^{\mathcal{G}}$ is non branching thus a simplicial pseudo manifold.

The link graphs of a GFT graph $\mathcal{G}$ are also GFT graphs (of lower dimensions). If $\mathcal{G}$ has no tadpole lines, none of its links can have tadpole lines (the lines of link graphs are descendants of lines of $\mathcal{G}$ ). The same reasoning as before holds for all the link graphs. Thus all the links of $\Delta^{\mathcal{G}}$ are also pseudo manifolds. Therefore $\Delta^{\mathcal{G}}$ is a normal pseudo manifold.

As a last remark, note that we used the fact that if a GFT graph is a simplicial complex then it has no tadpole line. If, however, a graph has 
no tadpole lines, its dual gluing might still not be simplicial complex: two vertices on a $n$ simplex could be identified after a longer sequence of gluings of lines (see section 4 for examples).

\section{Wrapping Singularities in GFT Graphs}

We will detail the singularities of GFT graphs in three dimensions. We will present several examples of three dimensional GFT graphs whose dual gluings do not respect proposition 1, namely

$$
\chi(\Delta) \neq|V(\Delta)|-\frac{1}{2} \sum_{i} \chi\left(\operatorname{lk}_{\Delta}\left(v_{i}\right)\right) .
$$

As the Euler characteristic is a topological invariant these gluings are not homeomorphic to pseudo manifolds. We will prove that whenever a GFT graph presents a certain type of singularity (we baptize wrapping singularity) it will not respect proposition 1. We will show that these singularities are generic (they appear at arbitrary high order in perturbations). These problems reappears in all higher dimensions, as similar singularities in the link graphs prevent any higher dimensional gluing from being a normal pseudo manifold.
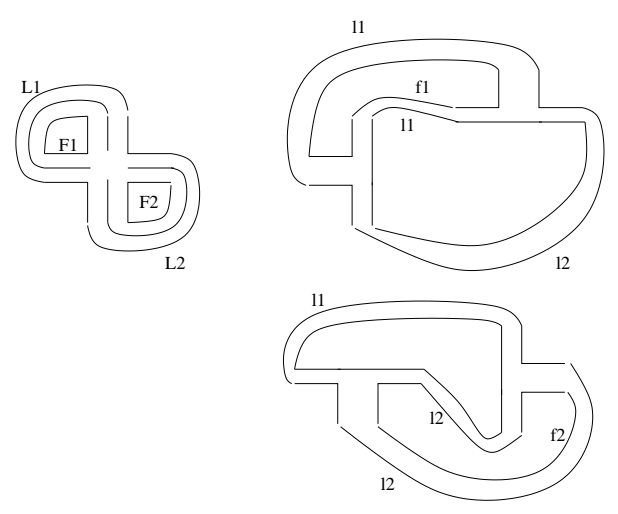

Figure 7: A GFT graph $\mathcal{G}^{1}$ dual to a gluing with negative Euler characteristic.

Consider the GFT graph $\mathcal{G}^{1}$ represented in figure 7. The lines applications are

$$
\begin{array}{ll}
l_{v_{A} v_{A}}^{(1)}(0)=1, & l_{v_{A} v_{A}}^{(1)}(1)=0, l_{v_{A} v_{A}}^{(1)}(2)=3, l_{v_{A} v_{A}}^{(1)}(3)=2, \\
l_{v_{A} v_{A}}^{(2)}(2)=3, & l_{v_{A} v_{A}}^{(2)}(0)=1, l_{v_{A} v_{A}}^{(2)}(1)=0, l_{v_{A} v_{A}}^{(2)}(3)=2,
\end{array}
$$


where, again, the first column presents the half lines identified by the lines $l_{v_{A} v_{A}}^{(1)}$ and $l_{v_{A} v_{A}}^{(2)}$. Denoting $A_{0}=A_{1}=\alpha, A_{2}=A_{3}=\beta$, the dual gluing writes

$$
\begin{aligned}
\Delta^{\mathcal{G}^{1}}= & \{\{\alpha, \alpha, \beta, \beta\},\{\alpha, \alpha, \beta\},\{\alpha, \beta, \beta\}, \\
& \{\alpha, \alpha\},\{\alpha, \beta\},\{\alpha, \beta\},\{\beta, \beta\},\{\alpha\},\{\beta\}, \emptyset\} .
\end{aligned}
$$

Note that the 3 simplex of this gluing is not a set, hence $\Delta^{\mathcal{G}^{1}}$ is not a simplicial complex. The Euler characteristic of $\Delta^{\mathcal{G}^{1}}$ is $\chi\left(\Delta^{\mathcal{G}^{1}}\right)=-1<0$ which breaks proposition 1. Therefore $\Delta^{\mathcal{G}^{1}}$ is a first example of a gluing not homeomorphic to a pseudo manifold.

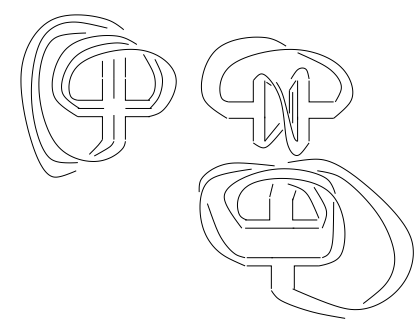

Figure 8: A second singular graph $\mathcal{G}^{2}$.

Proposition 1 fails again for the graph $\mathcal{G}^{2}$ of figure 8, which is in fact related by symmetry to $\mathcal{G}^{1}$. The Feynman amplitude of these graphs is

$$
A_{\mathcal{G}^{1}}=A_{\mathcal{G}^{2}}=\left[\delta^{\Lambda}(e)\right]^{2},
$$

where $\delta^{\Lambda}$ is a suitable cutoffed delta function on the group $G$, and $e$ is the identity element of $G$ (see [35, 36] for details on the computation of Feynman amplitudes in GFT).

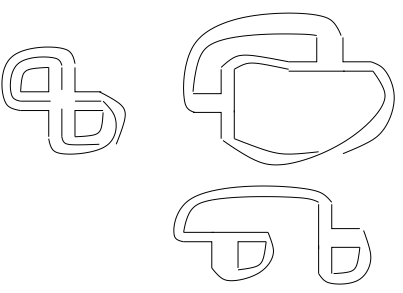

Figure 9: A third singular graph $\mathcal{G}^{3}$. 
The graph $\mathcal{G}^{3}$ in figure 9 has a planar link ( with Euler characteristic is 2) and a non orientable one (with Euler characteristic 1), hence $V$ $\frac{1}{2} \sum_{i} \chi\left(\operatorname{lk} v_{i}\right)=1 / 2$ which is not even an integer. Its amplitude is

$$
A_{\mathcal{G}^{3}}=\delta^{\Lambda}(e) .
$$

At first order one also has a GFT graph dual to a gluing homeomorphic to a pseudo manifold (in fact homeomorphic to the manifold $S^{3}$ ), presented in figure 10 .

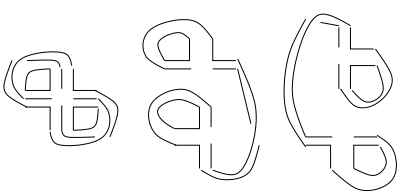

Figure 10: A graph $\mathcal{G}^{4}$ dual to a sphere $S^{3}$.

The reader should not be distracted by the twists of the lines in the link graphs: they can be undone by flipping either of the end vertices. We prefer to represent the twists explicitly so that the reader can easily identify the the descendant vertices in the link graphs. In detail the line applications are

$$
\begin{array}{ll}
\ell^{(1)}(0)=1 & \ell^{(1)}(3)=3, \ell^{(1)}(2)=2, \ell^{(1)}(1)=0, \\
\ell^{(2)}(2)=3 & \ell^{(2)}(1)=1, \ell^{(2)}(0)=0, \ell^{(2)}(3)=2 .
\end{array}
$$

The gluing dual to this graph writes (denoting $A_{0}=A_{1}=\alpha$ and $A_{2}=A_{3}=$ $\beta)$

$$
\begin{aligned}
\Delta^{\mathcal{G}^{3}}= & \{\{\alpha, \alpha, \beta, \beta\},\{\alpha, \alpha, \beta\},\{\alpha, \beta, \beta\}, \\
& \{\alpha, \alpha\},\{\alpha, \beta\},\{\beta, \beta\},\{\alpha\},\{\beta\}, \emptyset\} .,
\end{aligned}
$$

to be compared with eq. (27). This amplitude of $\mathcal{G}^{4}$ is

$$
A_{\mathcal{G}^{4}}=\delta^{\Lambda}(e) .
$$

The analysis of these first four examples of graphs leads to the flowing conclusions: 
- At first order, graphs not dual to pseudo manifolds are larger in power counting than graphs dual to pseudo manifolds. At arbitrary order, a graph obtained by star subdivisions ("one-four moves") of $\mathcal{G}^{1}$ will consistently have one extra power of $\delta_{\Lambda}(e)$ with respect to the similar graph obtained from $\mathcal{G}^{2}$.

- Restricting the permutations of strands allowed on the three dimensional GFT lines does not solve the problem: there exist singular graphs generated by even as well as odd permutations of the strands. Although (as we will see in the sequel) this idea is part of the solution, by itself it is insufficient.

As all the examples we presented so far exhibit tadpole lines, the reader might still hope that the singularities are just an artifact of these tadpoles. This is not true, the example of 11 presents a graph with no tadpole lines, whose links have Euler characteristics 1,2 and 2 , hence $V-\frac{1}{2} \sum_{i} \chi\left(\operatorname{lk} v_{i}\right)=\frac{1}{2}$ again.

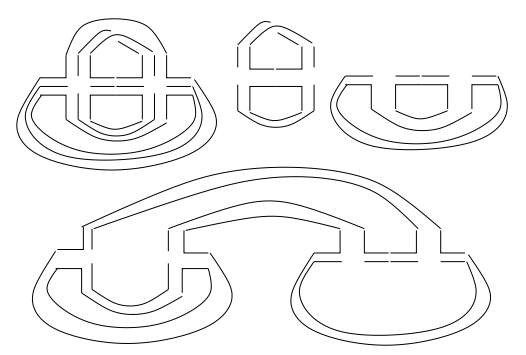

Figure 11: A singular graph $\mathcal{G}^{5}$ with no tadpole lines.

The proposition 11 fails for arbitrary GFT graphs because the detailed balance crucial for its proof

- each GFT vertex has four descendant vertices in the link graphs.

- each GFT line has three descendant lines in the link graphs.

- each GFT face has two descendant faces in the link graphs.

does not hold in general. The attentive reader will recall the correct balance encoded in eq. (23) and (24)

- each GFT vertex has four descendant vertices in the link graphs. 
- each GFT line has three descendant lines in the link graphs.

- each strand on a GFT vertex has two descendant strands in the link graphs.

The faces are closed strands, and in all the singular cases we presented the two descendant of some strand on a GFT vertex belong to only one face in the link graph. For instance in figure 7 we denoted $F_{1}$ and $F_{2}$ two GFT faces, and $f_{1}$ and $f_{2}$ their unique descendant faces in the link graphs. The faces of the link graphs $\left(f_{1}\right.$ and $\left.f_{2}\right)$ wrap twice around the GFT faces $\left(F_{1}\right.$ and $F_{2}$ ), hence the name "wrapping singularities". The reader can check that this phenomenon is present in all the examples we presented.

Whenever such singularities are present $\sum_{i} f^{0}\left(\operatorname{lk}_{\Delta \mathcal{G}}\left(v_{i}\right)\right)<2 f^{1}\left(\Delta^{\mathcal{G}}\right)$ and $\Delta^{\mathcal{G}}$ does not respect proposition 1 hence it is not homeomorphic to a pseudo manifold. The wrapping singularities are generic in GFT: a graph having subgraphs like the ones in figure 12, 13 will have a wrapping singularity.

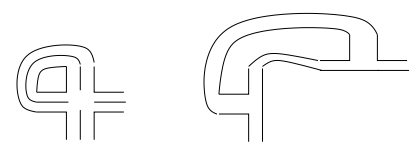

Figure 12: A subgraph leading to a wrapping singularity.

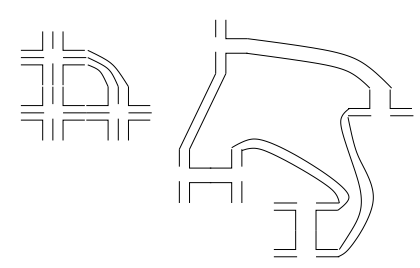

Figure 13: A second subgraph leading to a wrapping singularity.

In figure 14 we give an example of a four dimensional graph having a wrapping singularity. The face $F_{01}$ has only two three dimensional descendants (instead of three), denoted both $f_{01}$, and one of them wraps twice around $F_{01}$.

The situation looks bleak for GFT's. The graphs with wrapping singularities are large in power counting and generic. Singular graphs dominate the "low energy" effective behavior of GFT's and render them unsatisfactory. 


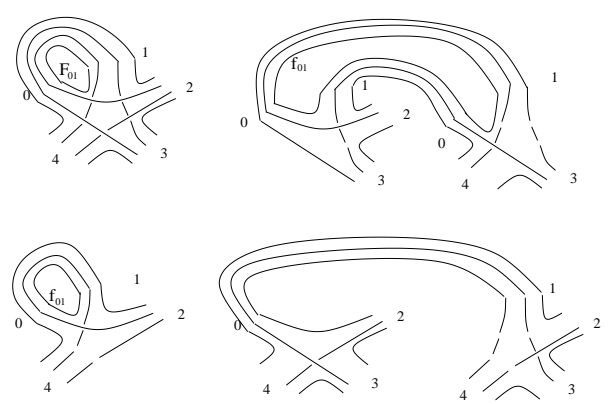

Figure 14: A four dimensional graph having a wrapping singularity.

\section{The Colored GFT Graphs and Pseudo Ma- nifolds}

In this section we prove that colored GFT (CGFT) model [1] completely solves the problem of non pseudo manifold graphs in a single stroke, in arbitrary dimension. By coloring our quantum field we introduce a combinatorial constraint in all its graphs and completely eliminate the wrapping singularities. Moreover, once the combinatorial constraints are properly understood the proof that all CGFT graphs are dual to normal pseudo manifolds is practically tautological. For this reason the CGFT model is, in our opinion, the appropriate GFT model one should always consider when treating GFT's as quantum field theories.

In $n$ dimensions, the colored GFT model is defined by $n+1$ pairs of fermionic (or complex bosonic) fields $\psi^{p}, \bar{\psi}^{p}: G^{n} \rightarrow \mathbb{G}$ or $\mathbb{C}$, invariant under left group multiplication of the argument, and with no symmetry properties. The action of the colored GFT writes

$$
\begin{aligned}
S= & \frac{1}{2} \int[d g] \sum_{p=0}^{n} \psi_{\alpha_{0} \alpha_{1} \ldots \alpha_{n}}^{p} \bar{\psi}_{\alpha_{0} \alpha_{1} \ldots \alpha_{n}}^{p}+S_{i n t}+\bar{S}_{i n t}, \\
S_{i n t}= & \lambda \int[d g] \psi_{\alpha_{0 n} \alpha_{0 n-1} \ldots \alpha_{01}}^{0} \ldots \psi_{\alpha_{p p-1} \ldots \alpha_{p 0} \alpha_{p n} \ldots \alpha_{p p+1}}^{p} \\
& \times \ldots \psi_{\alpha_{n n-1} \ldots \alpha_{n 0}}^{n},
\end{aligned}
$$

and $\bar{S}_{\text {int }}$ has the same form as $S_{\text {int }}$ with $\psi$ replaced by $\bar{\psi}$. The index $p$ on each field is a color index and we denote the set of all colors $\mathcal{C}^{n+1}=\{0, \ldots, n\}$.

The interaction part of the colored GFT model has two terms and generates two vertices: the positive vertex, involving only $\psi$ 's, represented in figure 
3 (where the labels $0, \ldots, n$ become now colors), and the negative vertex, involving only $\bar{\psi}$, with colors turning anticlockwise around it. The propagator of the model has $n$ parallel strands and always connects two half lines of the same color, one on a positive and one on a negative vertex. We orient all lines from positive to negative vertices.

The strand structure of the vertex and propagator is rigid thus a CGFT graph admits a simplified representation as a colored graph. The colored graph is obtained by collapsing all the strands of the lines in "thin" lines, and all the strands of the vertices in point vertices. Conversely, given a colored graph with thin lines and point vertices one can reconstruct the stranded graph associated to it. Figure 15 depicts a CGFT graph either as a stranded graph (on the left) or as colored graph (on the right).
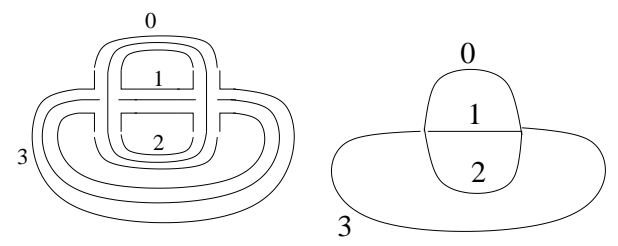

Figure 15: The unique second order CGFT graph $\mathcal{G}$.

A CGFT graph $\mathcal{G}$ comes equipped with a natural family of subgraphs, called the $p$-bubbles. A $p$-bubble is a connected subgraph of $\mathcal{G}$ made only of lines of colors in $\mathcal{C}^{p}$ for some subset $\mathcal{C}^{p} \subset \mathcal{C}^{n+1}$ of cardinality $\left|\mathcal{C}^{p}\right|=p$. We denote a $p$-bubble with colors $\mathcal{C}^{p}$ and vertices $\mathcal{V}$ by $\mathcal{B}_{\mathcal{V}}{ }^{p}$.

Clearly the 0 -bubbles of a graph are its vertices and the 1-bubbles are its lines. For $p \geq 2$ the $p$-bubbles admit two graphical representations, either as colored graphs or as stranded graphs. In the stranded graph representation one only draws the strands common to the lines of colors $\mathcal{C}^{p}$. The colored and stranded representation of the 3-bubbles of the graph $\mathcal{G}$ in figure 15 are depicted in figure 16.

The $p$-bubbles are themselves colored GFT graphs in $(p-1)$ dimensions. Comparing figure 16 with figure 6, we note that for this graph the 3-bubbles correspond to the link graphs. This is in fact a general result for $p \geq 2$

Theorem 1. For $p \geq 2$, the $p$-bubbles of a CGFT graph are the link graphs of the $(n-p)$ simplices in the gluing $\Delta^{\mathcal{G}}$.

Proof: Consider two vertices $v_{A}$ (positive) and $v_{B}$ (negative) connected by a line of color $i$ (see figure 17) in an $n$-dimensional GFT graph $\mathcal{G}$. 

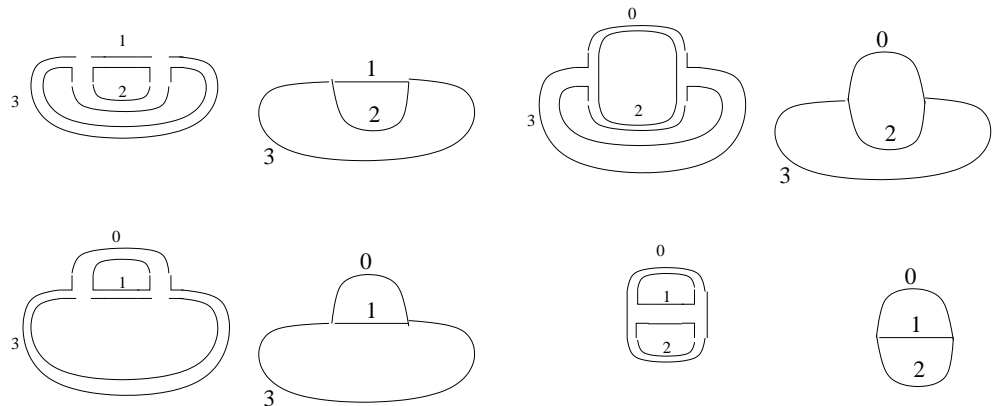

Figure 16: The 3-bubbles of $\mathcal{G}$ represented as stranded or as colored graphs.

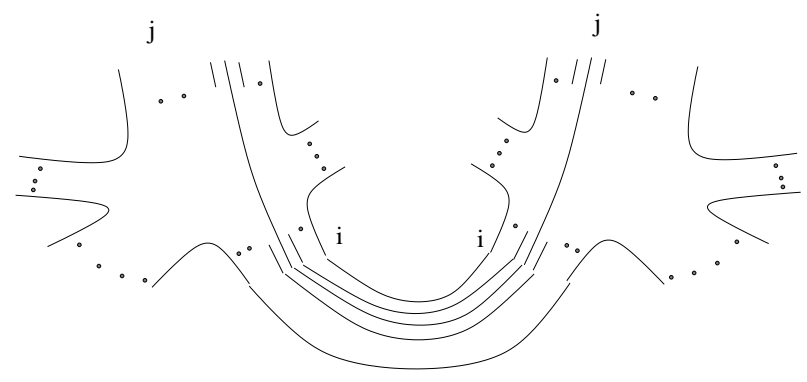

Figure 17: A colored GFT line.

This drawing essentially proves the result. As the line has only parallel strands and connects opposite vertices, the strand $j i$, common to the half lines $j$ and $i$ on $v_{A}$ necessarily connects with the strand $i j$ on $v_{B}$. This holds for all lines, therefore the labels $i j$ are conserved all along the strand. This is the fundamental difference between the usual GFT graphs and the CGFT graphs and render the latter much better behaved.

The vertex $v_{A}$ is dual to some simplex $\sigma^{n}=\left\{A_{0}, \ldots A_{n}\right\}$. Consider one of its $(n-p)$ subsimplices

$$
\sigma^{n-p}=\left\{A_{0}, \ldots, A_{n}\right\} \backslash\left\{A_{i_{1}}, \ldots, A_{i_{p}}\right\}
$$

Following subsection 3.1, the contribution of $v_{A}$ to the link graph of $\sigma^{p}$ is the $p$ dimensional GFT vertex (descendant of $v_{A}$ ) with labels $\left(i_{1} \ldots i_{p}\right)$. But, as the colors of strands are conserved, this vertex will always connect only with the link vertex $\left(i_{1} \ldots i_{p}\right)$ descending from $v_{B}$.

The link graphs are exactly the connected $p$ dimensional GFT graphs formed by lines and strands with colors $\left\{i_{1}, \ldots i_{p}\right\}$, hence the $p$ bubbles of $\mathcal{G}$. 
We are now in the position to state and prove the core result of this paper.

Theorem 2. Any connected $n$ dimensional CGFT graph is dual to a normal simplicial pseudo manifold.

Proof By lemma 1 it is enough to prove that the gluing $\Delta^{\mathcal{G}}$ dual to any CGFT graph is a simplicial complex. This is trivial once the appropriate notations are introduced.

The 0 simplices (vertices) of the gluing $\Delta^{\mathcal{G}}$ are dual to the $n$-bubbles of the CGFT graph, $V\left(\Delta^{\mathcal{G}}\right)=\left\{\mathcal{B}_{\mathcal{V}}^{\mathcal{C}^{n+1} \backslash\{p\}}\right\}$.

The 1 simplices of $\Delta^{\mathcal{G}}$ the $(n-1)$-bubbles of $\mathcal{G}$. Consider one of the $(n-1)$ bubbles of $\mathcal{G}$, say $\mathcal{B}_{\mathcal{V}}^{\mathcal{C}^{n+1} \backslash\{p, q\}}$. This bubble is subgraph of $\mathcal{G}$, hence there exists an unique subgraph obtained by adding all the lines of color $p$ for incident on the vertices $\mathcal{V}$ and then closing the entire connected component with colors $\mathcal{C}^{n+1} \backslash\{q\}$, that is

$$
\forall \mathcal{B}_{\mathcal{V}}^{\mathcal{C}^{n+1} \backslash\{p, q\}}, \exists ! \mathcal{B}_{\mathcal{V}^{\widehat{q}}}^{\mathcal{C}^{n+1} \backslash\{q\}}, \mathcal{V} \subset \mathcal{V}^{\widehat{q}}
$$

where we denoted $\mathcal{V}^{\widehat{q}}$ the (unique) maximal set of vertices connected by lines of colors $\mathcal{C}^{n+1} \backslash\{q\}$ and containing $\mathcal{V}$. The same holds for the lines of color $p$. Pick any vertex $v_{A} \in \mathcal{V}$, dual to a $n$ simplex $\left\{A_{0}, \ldots A_{n}\right\}$. The 1 simplex dual to $\mathcal{B}_{\mathcal{V}}^{\mathcal{C}^{n+1} \backslash\{p, q\}}$ is $\left\{A_{p}, A_{q}\right\}$, and the 0 simplex dual to $\mathcal{B}_{\mathcal{V}^{\widehat{q}}}^{\mathcal{C}^{n+1} \backslash\{q\}}$ is $\left\{A_{q}\right\}$. Therefore the 1 simplex dual to $\mathcal{B}_{\mathcal{V}}^{\mathcal{C}^{n+1} \backslash\{p, q\}}$ writes

$$
\left\{\mathcal{B}_{\mathcal{V}^{\widehat{q}}}^{\mathcal{C}^{n+1} \backslash\{q\}}, \mathcal{B}_{\mathcal{V}^{\widehat{p}}}^{\mathcal{C}^{n+1} \backslash\{p\}}\right\}, \quad \mathcal{V} \subset \mathcal{V}^{\widehat{q}} \cap \mathcal{V}^{\widehat{p}}
$$

Similarly, a $p$ simplex $\sigma^{p}$ is dual to a $(n-p)$ bubble $\mathcal{B}_{\mathcal{V}}^{\mathcal{C}^{n} \backslash\left\{i_{0}, \ldots i_{p}\right\}}$, and for each $(n-p)$ bubble and each color $i_{q}$, there exists an unique subgraph with $n$ colors obtained by adding the lines of colors all colors except $i_{q}$ incident on the vertices $\mathcal{V}$ and completing the connected component with lines of all colors except $i_{q}$ (whose set of vertices we denote $\widehat{\mathcal{V}^{\left(i_{q}\right.}}$ )

$$
\mathcal{B}_{\mathcal{V}^{\hat{i_{q}}}}^{\mathcal{C}^{n+1} \backslash\left\{i_{q}\right\}}, \quad \mathcal{V} \subset \mathcal{V}^{\hat{i_{q}}}
$$

The $p$ simplex $\sigma^{p}$ writes

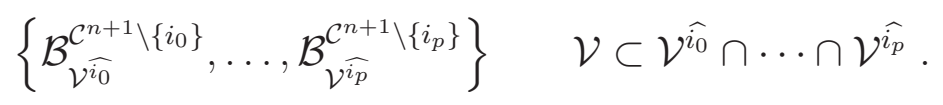


The proof is now tautological. For any subset $M^{k} \subset \sigma^{p}$ of cardinality $(k+1)<(p+1)$,

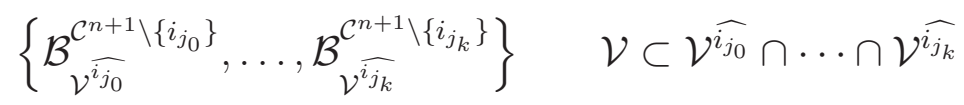

with $\left\{j_{0}, \ldots j_{k}\right\} \subset\{0, \ldots p\}$ there exists a $k$-bubble obtained by adding all lines of colors $\{0, \ldots p\} \backslash\left\{j_{0}, \ldots j_{k}\right\}$ to $\mathcal{V}$ and completing the graph thus obtained to a bubble of colors $\mathcal{C}^{n+1} \backslash\left[\left\{i_{0} \ldots i_{p}\right\} \backslash\left\{i_{j_{0}} \ldots i_{j_{k}}\right\}\right]$. Consequently $M^{k}$ is the simplex dual to this bubble, $M^{k} \in \Delta^{\mathcal{G}}$, and the gluing is a simplicial complex.

In retrospect one sees that all the link graphs are orientable, as they are allways made of colored lines joining vertices of opposite orientation. The colored GFT model is the simplest one which guarantees that all sub simplices, of arbitrary dimension, are allways identified coherently in all gluings corresponding to the CGFT lines.

\section{Conclusion}

We started out work by a in depth study of singularities in the usual GFT models. We concluded that highly pathological GFT graphs whose dual gluings are not homeomorphic to pseudo manifolds dominate in power counting and proliferate in the perturbative development of the usual GFT's. Of course, as long as one analyzes particular examples of "nice" graphs one is oblivious to this problem. However, the moment one tries to treat the usual GFT's as fully fledged quantum field theories and take into account all the graphs, the pathological ones dominate. In order to save the GFT's as quantum field theories and obtain a reasonable effective behavior one must deal one way or another with this problem.

The solution we present in this paper is to use the Colored GFT models. The extra structure encoded in the coloring eliminates the wrapping singularities for all graph and in all dimensions in a very natural way.

A large amount of work still remains to be done before establishing the colored GFT's as quantum field theories, most importantly one would like to find some scaling regime in which their effective behavior is dominated by manifold configurations. On the other hand the language of the colored GFT's could be used as a mathematical tool to further the understanding of 
topology. The encoding of the link graphs into the bubbles provides a bridge between topology and combinatorics opening up the possibility to obtain, using the latter, new results concerning the former.

\section{Acknowledgements}

Research at Perimeter Institute is supported by the Government of Canada through Industry Canada and by the Province of Ontario through the Ministry of Research and Innovation.

\section{A Remarks on Simplicial Complexes}

Remark 1. The collection $\operatorname{star}_{\Delta}(v) \backslash l k_{\Delta}(v)$ is

$$
\operatorname{star}_{\Delta}(v) \backslash l k_{\Delta}(v)=\left\{\{v\} \cup \tau \mid \tau \in l k_{\Delta}(v)\right\} .
$$

Proof: " $\supset$ ": Let any $\tau \in \mathrm{lk}_{\Delta}(v)$. Then $\{v\} \cup \tau \in \Delta$ and $\{v\} \cup \tau \in \operatorname{star}_{\Delta}(v)$. But, as $\{v\} \in\{v\} \cup \tau$ then $\{v\} \cup \tau \notin \mathrm{lk}_{\Delta}(v)$, thus $\{v\} \cup \tau \in \operatorname{star}_{\Delta}(v) \backslash \operatorname{lk}_{\Delta}(v)$.

" $\subset$ ": Let $\sigma \in \operatorname{star}_{\Delta}(v) \backslash \mathrm{lk}_{\Delta}(v)$. Then $\sigma \cup\{v\} \in \Delta$ and $v \in \sigma$, thus $\sigma \cup\{v\}=\sigma \in \Delta$. Denote $\tau=\sigma \backslash\{v\}$. As $\sigma \in \Delta$, and $\Delta$ is a simplicial complex, $\tau \in \Delta$. Therefore $\tau \in \Delta, \tau \cap\{v\}=\emptyset$ and $\tau \cup\{v\}=\sigma \in \Delta$, hence $\tau \in \mathrm{lk}_{\Delta}(v)$.

Remark 2. Let $\Delta$ a simplicial complex with simplices of maximal dimension $n$. For any vertex $v$ of $\Delta$

$$
\chi\left(\operatorname{star}_{\Delta}(v)\right)=1 .
$$

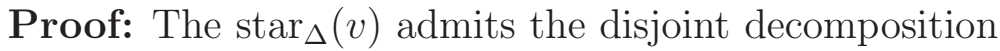

$$
\operatorname{star}_{\Delta}(v)=\left(\operatorname{star}_{\Delta}(v) \backslash \mathrm{lk}_{\Delta}(v)\right) \cup \mathrm{lk}_{\Delta}(v) .
$$

Under this decomposition all simplices either belong to $\operatorname{star}_{\Delta}(v) \backslash \mathrm{lk}_{\Delta}(v)$ or to $\operatorname{lk}_{\Delta}(v)$.

Let any $p \geq 0$ simplex in the star, $\sigma^{p}$. Either $\sigma^{p} \in \mathrm{lk}_{\Delta}(v)$ or $\sigma^{p}=$ $\{v\} \cup \sigma^{p-1}, \sigma^{p-1} \in \mathrm{lk}_{\Delta}(v)$. Note that by definition the link has exactly one -1 simplex, namely $\emptyset$, and zero $n$ simplices. 
Denote the number of $p$ simplices in the star $N_{p}$, and the number of $p$ simplices in the link $n_{p}$. Then

$$
f^{p}\left(\operatorname{star}_{\Delta}(v)\right)=f^{p}\left(\mathrm{lk}_{\Delta}(v)\right)+f^{p-1}\left(\mathrm{lk}_{\Delta}(v)\right)
$$

hence,

$$
\begin{aligned}
& \chi\left(\operatorname{star}_{\Delta}(v)\right)=\sum_{p=0}^{n}(-)^{n} f^{p}\left(\operatorname{star}_{\Delta}(v)\right) \\
& =\sum_{p=0}^{p}(-)^{p}\left(f^{p}\left(\mathrm{lk}_{\Delta}(v)\right)+f^{p-1}\left(\mathrm{lk}_{\Delta}(v)\right)\right)=f^{-1}\left(\mathrm{lk}_{\Delta}(v)\right)=1 .
\end{aligned}
$$

Remark 3. The links of a simplicial pseudo manifold $\Delta$ are pure and non branching.

Proof: Consider a simplex $\tau^{p}$ of dimension $p$ in a simplicial pseudo manifold and denote $\sigma_{(1)}^{n}, \ldots \sigma_{(N)}^{n}$ all the $\mathrm{n}$ dimensional simplices to which $\tau^{p}$ is a face. Denote also $\sigma_{(k)}^{n-p-1}=\sigma_{(k)}^{n} \backslash \tau^{p}$.

Step 1: $\operatorname{lk}_{\Delta}\left(\tau^{p}\right)$ is a $(\mathrm{n}-\mathrm{p}-1)$ - dimensional pure simplicial complex.

Any $\sigma \in \mathrm{lk}_{\Delta}\left(\tau^{p}\right)$ one has $\sigma \cup \tau \in \Delta$ hence there exists some $\mathrm{n}$ dimensional simplex $\rho^{n} \supseteq \sigma \cup \tau$. But $\tau \subseteq \rho^{n}$ thus $\rho^{n}=\sigma_{(k)}^{n}$ for some $k$, and $\sigma \subseteq \sigma_{(k)}^{n} \backslash \tau^{p}=$ $\sigma_{(k)}^{n-p-1}$.

Step 2: $\mathrm{lk}_{\Delta}\left(\tau^{p}\right)$ is non branching.

Let $\sigma^{n-p-2}$ a $(n-p-2)$ simplex in $\operatorname{lk}_{\Delta}\left(\tau^{p}\right)$. It is a face of $r$ simplices of dimension $(n-p-1), \sigma_{\left(k_{1}\right)}^{n-p-1}, \ldots \sigma_{\left(k_{r}\right)}^{n-p-1}$. Then $\tau^{p} \cup \sigma^{n-p-2}$ is a $n-1$ dimensional simplex of $\Delta$ which is a face of the $r$ simplices $\tau^{p} \cup \sigma_{k_{i}}^{n-p-1}$ of dimension $n$. As $\Delta$ is non branching, $r=2$ hence $\operatorname{lk}_{\Delta}\left(\tau^{p}\right)$ is non branching.

However the links of simplicial pseudo manifolds are not necessarily strongly connected. For example, in the simplicial pseudo manifold

$$
\begin{array}{r}
\left\{\left\{v, a_{1}, a_{2}\right\},\left\{v, a_{1}, a_{3}\right\},\left\{v, a_{2}, a_{3}\right\},\left\{v, b_{1}, b_{2}\right\},\left\{v, b_{1}, b_{3}\right\},\left\{v, b_{2}, b_{3}\right\}\right. \\
\left\{b_{1}, b_{2}, m_{12}\right\},\left\{a_{1}, a_{2}, m_{12}\right\},\left\{b_{1}, a_{1}, m_{12}\right\},\left\{b_{2}, a_{2}, m_{12}\right\} \\
\left\{b_{1}, b_{3}, m_{13}\right\},\left\{a_{1}, a_{3}, m_{13}\right\},\left\{b_{1}, a_{1}, m_{13}\right\},\left\{b_{3}, a_{3}, m_{13}\right\} \\
\left.\left\{b_{2}, b_{3}, m_{23}\right\},\left\{a_{2}, a_{3}, m_{23}\right\},\left\{b_{2}, a_{2}, m_{23}\right\},\left\{b_{3}, a_{3}, m_{23}\right\}\right\},
\end{array}
$$

the link of $v$ is not even connected, much less strongly connected. 


\section{References}

[1] R. Gurau, arXiv:0907.2582 [hep-th]].

[2] D. V. Boulatov, Mod. Phys. Lett. A 7, 1629 (1992) arXiv:hep-th/9202074.

[3] L. Freidel, Int. J. Theor. Phys. 44, 1769 (2005) [arXiv:hep-th/0505016].

[4] M. Gross, Nucl. Phys. Proc. Suppl. 25A, 144 (1992).

[5] J. Ambjorn, B. Durhuus and T. Jonsson, Mod. Phys. Lett. A 6, 1133 (1991).

[6] N. Sasakura, Mod. Phys. Lett. A 6, 2613 (1991).

[7] N. Sasakura, arXiv:1005.3088 [hep-th]].

[8] R. Williams, in [11]

[9] J. Ambjorn, J. Jurkiewicz and R. Loll, Phys. Rev. D 72, 064014 (2005) arXiv:hep-th/0505154.

[10] D. Oriti, Rept. Prog. Phys. 64, 1489 (2001) arXiv:gr-qc/0106091.

[11] D. Oriti, ed., Approaches to Quantum Gravity: toward a new understanding of space, time and matter, Cambridge University Press, Cambridge (2009)

[12] F. David, Nucl. Phys. B 257, 543 (1985).

[13] D. Oriti, in Quantum Gravity, B. Fauser, J. Tolksdorf and E. Zeidler, eds., Birkhaeuser, Basel, (2007), [arXiv: gr-qc/0512103]

[14] J. W. Barrett and I. Naish-Guzman, Class. Quant. Grav. 26, 155014 (2009) arXiv:0803.3319 [gr-qc]].

[15] V. G. Turaev and O. Y. Viro, Topology 31, 865 (1992).

[16] A. Abdesselam, Sém. Lothar. Combin. 49 (2002/04), Art. B49c, 45 pp. (electronic). arXiv:math/0212121.

[17] A. Abdesselam, arXiv:0904.1734v2[math.GT]]. 
[18] J. Engle, R. Pereira and C. Rovelli, Phys. Rev. Lett. 99, 161301 (2007) arXiv:0705.2388 [gr-qc]].

[19] J. Engle, R. Pereira and C. Rovelli, Nucl. Phys. B 798, 251 (2008) arXiv:0708.1236 [gr-qc]].

[20] E. R. Livine and S. Speziale, Phys. Rev. D 76, 084028 (2007) arXiv:0705.0674 [gr-qc]].

[21] L. Freidel and K. Krasnov, Class. Quant. Grav. 25, 125018 (2008) arXiv:0708.1595 [gr-qc]].

[22] F. Conrady and L. Freidel, Phys. Rev. D 78, 104023 (2008) arXiv:0809.2280 [gr-qc]].

[23] V. Bonzom, E. R. Livine, M. Smerlak and S. Speziale, Nucl. Phys. B 804, 507 (2008) arXiv:0802.3983 [gr-qc]].

[24] D. Oriti and T. Tlas, Class. Quant. Grav. 27, 135018 (2010) arXiv:0912.1546 [gr-qc]].

[25] A. Baratin and D. Oriti, arXiv:1002.4723 [hep-th]].

[26] D. Oriti, arXiv:0912.2441 [hep-th]].

[27] W. J. Fairbairn and E. R. Livine, Class. Quant. Grav. 24, 5277 (2007) arXiv:gr-qc/0702125].

[28] A. Di Mare and D. Oriti, arXiv:1001.2702 [gr-qc]].

[29] A. Ashtekar, M. Campiglia and A. Henderson, Phys. Lett. B 681, 347 (2009) [arXiv:0909.4221 [gr-qc]].

[30] A. Ashtekar, M. Campiglia and A. Henderson, Class. Quant. Grav. 27, 135020 (2010) [arXiv:1001.5147 [gr-qc]].

[31] H. Grosse and R. Wulkenhaar, Commun. Math. Phys. 256, 305 (2005) arXiv:hep-th/0401128.

[32] R. Gurau, J. Magnen, V. Rivasseau and F. Vignes-Tourneret, Commun. Math. Phys. 267, 515 (2006) arXiv:hep-th/0512271. 
[33] M. Disertori, R. Gurau, J. Magnen and V. Rivasseau, Phys. Lett. B 649, 95 (2007) arXiv:hep-th/0612251.

[34] J. B. Geloun, R. Gurau and V. Rivasseau, Phys. Lett. B 671, 284 (2009) arXiv:0805.4362 [hep-th]].

[35] L. Freidel, R. Gurau and D. Oriti, Phys. Rev. D 80, 044007 (2009) arXiv:0905.3772 [hep-th]].

[36] J. Magnen, K. Noui, V. Rivasseau and M. Smerlak, Class. Quant. Grav. 26, 185012 (2009) arXiv:0906.5477 [hep-th]].

[37] J. B. Geloun, J. Magnen and V. Rivasseau, arXiv:0911.1719 [hep-th]].

[38] J. B. Geloun, T. Krajewski, J. Magnen and V. Rivasseau, arXiv:1002.3592 [hep-th]].

[39] R. Gurau, arXiv:0911.1945 [hep-th]].

[40] V. Bonzom and M. Smerlak, arXiv:1004.5196 [gr-qc]].

[41] R. De Pietri and C. Petronio, J. Math. Phys. 41, 6671 (2000) arXiv:gr-qc/0004045.

[42] S. Alexander, L. Crane and M. D. Sheppeard, arXiv:gr-qc/0306079.

[43] Dmitry Kozlov, Combinatorial Algebraic Topology, Springer, ISBN-10: 354071961X, ISBN-13: 978-3540719618 